\title{
Reforma Sanitária Brasileira e políticas farmacêuticas: uma análise dos fatos produzidos entre 2003 e 2014
}

\author{
Brazilian Health Reform and pharmaceutical policies: an analysis of \\ the facts produced between 2003 and 2014
}

Tatiane de Oliveira Silva Alencar1, Jairnilson Silva Paim²

RESUMO Estudo de caso cujo objetivo é analisar as políticas farmacêuticas do período de 2003 a 2014, tendo como referência a Reforma Sanitária Brasileira. As fontes de evidência foram documentos e entrevistas semiestruturadas, e o processamento dos dados ocorreu mediante a técnica da análise de conteúdo temática. Os dados obtidos foram organizados em dois momentos (períodos Lula e Dilma) e a análise foi fundamentada na teoria marxista. Os resultados indicam que a instituição das políticas farmacêuticas foi favorecida pela participação de atores sociais vinculados ao movimento sanitário no âmbito do Estado, o que permitiu uma reestruturação dessa agenda, ainda que mediante conflitos.

PALAVRAS-CHAVE Reforma dos serviços da saúde. Política de saúde. Medicamentos. Sistema Único de Saúde.

ABSTRACT This is a case study whose objective is to analyze pharmaceutical policies of the period from 2003 to 2014, having as reference the Brazilian Health Reform. The sources of evidence were semi-structured interviews and documents, and the data processing was performed using the thematic content analysis technique. The data obtained were organized in two moments (Lula and Dilma periods) and the analysis was based on Marxist theory. The results indicate that the institution of pharmaceutical policies was favored by the participation of social actors linked to the health movement within the State, which has permitted a restructuring of this agenda, even through conflicts.

KEYWORDS Health care reform. Health policy. Medicine. Unified Health System.

1 Universidade Estadual de Feira de Santana (UEFS)

- Feira de Santana (BA), Brasil.

tatifarmauefs@yahoo.com.br

2 Universidade Federal da Bahia (UFBA), Instituto de Saúde Coletiva (ISC) Salvador (BA), Brasil. jairnil@ufba.br 


\section{Introdução}

Entende-se uma política farmacêutica como uma política de saúde, ou seja, uma decisão (ação ou omissão) do Estado (TEIXEIRA; PAIM 2005), em relação às questões relativas a medicamentos e à assistência farmacêutica. Em todo o mundo, o debate acerca destas questões é intenso e tem acompanhado as discussões da reforma dos sistemas de saúde. No Brasil, sempre se adotou alguma ação para atender à necessidade de medicamentos manifestada pela sociedade ou por parte dela, tais como: a implantação e o desenvolvimento dos laboratórios oficiais; a criação (1971) e a extinção (1997) da Central de Medicamentos; o reconhecimento (ou não) de patentes de produtos farmacêuticos, nos diferentes governos; a regulação da propaganda de medicamentos; e medidas para a ampliação do acesso.

Apesar desses antecedentes, a Política Nacional de Medicamentos (PNM), instituída em 1998, é considerada em muitos estudos como marco das políticas farmacêuticas, pois se refere ao primeiro documento oficial, após a instituição do Sistema Único de Saúde (SUS), relativo à questão de medicamentos. Além da PNM, aspectos da formulação e da implantação de políticas farmacêuticas, da disponibilidade de medicamentos em diferentes regiões do Brasil, da avaliação e da organização da assistência farmacêutica, do financiamento e da judicialização para a garantia do acesso foram analisados em diversos estudos, conforme identificado em bases nacionais e internacionais de periódicos, dissertações e teses. Não se identificaram estudos, além da tese que deu origem a este artigo, que tenham abordado tais políticas sob o referencial da Reforma Sanitária Brasileira (RSB). Daí, a pertinência de serem examinados os fatos produzidos entre 2003 e 2014 com este foco.

Nesteparticular,ostermos 'medicamentos' e 'assistência farmacêutica' apresentam concepções técnicas e políticas distintas, tendo em vista o processo de constituição das políticas farmacêuticas nos diferentes momentos históricos. Tomando como referência a década de 1970, quando se fortalece o movimento pela RSB, o debate inicial enfatizou a necessidade de garantir a disponibilidade de fármacos e, desse modo, que o Estado instituísse uma política de medicamentos (CORDEIRO, 1978). Posteriormente, esse debate se ampliou e se articulou ao movimento das entidades farmacêuticas para a incorporação de práticas profissionais mais coerentes aos princípios e diretrizes do SUS, em discussão naquele momento. Assim, emergiu a demanda por uma política de assistência farmacêutica que articulasse as dimensões tecnológica, sanitária, econômica, política, simbólica e ideológica do medicamento, em sua inserção no ciclo produtivo-econômico da saúde (ALENCAR, 2016).

Nesse processo, tem havido intensos conflitos entre o Estado, a sociedade civil e o mercado farmacêutico, especialmente multinacional, conforme demonstram estudos publicados desde os anos 1960 (LEDOGAR; KUCINSKI, 1977; PACHECO, 1968) evidenciando embates no âmbito do poder Legislativo, motivando a realização de seis Comissões Parlamentares de Inquéritos (CPI), que discutiram acerca da questão de medicamentos.

Considerando que a constituição de políticas farmacêuticas remete a um processo de produção social de alta complexidade, envolvendo relações e estratégias entre atores (MATUS, 2005), e entendendo o fato político como um fato social produzido a partir da interação de atores sociais com distintas capacidades acumuladas, tendo em vista o Estado (ALENCAR, 2016), o objetivo deste artigo é analisar os fatos políticos relativos às políticas farmacêuticas, no período de 2003 a 2014, identificando os atores envolvidos, os conflitos e 
as estratégias utilizadas, tendo como referência a RSB.

\section{Metodologia e referencial teórico}

Este artigo é parte dos resultados de uma pesquisa, que teve como objetivo analisar as possíveis relações da RSB com a questão Medicamentos/Assistência Farmacêutica (questão MAF) (ALENCAR, 2016). Originalmente, tratou-se de um estudo de caso, que teve como recorte temporal o período de 1976 a 2014. O presente trabalho delimita a análise de fatos políticos, relativos às políticas farmacêuticas, produzidos no período de 2003 a 2014, correspondendo aos períodos dos governos do presidente Luiz Inácio Lula da Silva - Lula (2003-2010) e da presidente Dilma Vana Rousseff (2011-2014).

Para a produção dos dados, foram utilizadas, como fontes de evidência, documentos e entrevistas semiestruturadas, com 22 informantes envolvidos com o movimento da RSB e/ou a questão MAF. As entrevistas foram guiadas por um roteiro pré-definido e os informantes consentiram na divulgação dos seus nomes. O estudo foi aprovado pelo Comitê de Ética em Pesquisa (parecer 690.819). Para o processamento do material empírico, utilizou-se a técnica da análise de conteúdo temática (BARDIN, 2011).

A análise dos dados foi orientada pelos elementos teóricos que fundamentaram o estudo, tendo sido considerados: momentos de análise da RSB trazidos por Paim (2008), contribuições da teoria marxista para entender o medicamento como um produto do ciclo produtivo-econômico da saúde e como uma necessidade social e existencial (HELLER, 1986; MARX, 1996), contribuições gramscianas (COUTINHO, 2011) para compreender o Estado e suas relações com a sociedade e o mercado, e as definições de poder, política, estratégia e ator social trazidas por Mário Testa $(1995,2005)$.

\section{Resultados}

\section{Período Lula (2003-2010)}

\section{CIÊNCIA, TECNOLOGIA E INOVAC̣ÃO EM SAÚDE, NA AGENDA DO PAÍS}

A criação da Secretaria de Ciência, Tecnologia e Insumos Estratégicos (SCTIE), em 2003, correspondeu ao atendimento de uma necessidade da área de Ciência e Tecnologia (C\&T), já expressada no projeto da RSB, no relatório da VIII Conferência Nacional de Saúde (PAIM, 2008), prevista na Constituição Federal de 1988 e na Lei Orgânica da Saúde (no 8.080/1990), e retomada em 1994, na I Conferência Nacional de Ciência e Tecnologia em Saúde (CNCTS), e nas Conferências Nacionais de Saúde (CNS) - X, XI e XII -, conforme evidenciado nos relatórios desses eventos. Assim, a articulação das pautas relativas a medicamentos, à assistência farmacêutica e à C\&T em um único órgão favoreceu uma maior organicidade a estas, na agenda política do País.

A proposta de uma secretaria específica contou com a participação de atores vinculados ao movimento sanitário - representantes da Associação Brasileira de Saúde Coletiva (Abrasco) e da Fundação Oswaldo Cruz (Fiocruz) - e à questão MAF - representantes da Federação Nacional de Farmacêuticos (Fenafar), a exemplo de Jorge Bermudez, ator relevante no processo de construção das políticas farmacêuticas; Jandira Feghali, médica, deputada federal (Partido Comunista do Brasil/RJ - PC do B-RJ); e José Alberto Hermógenes, médico sanitarista com participação ativa na construção do SUS. Este último, juntamente com o farmacêutico e militante de organizações farmacêuticas, Norberto Rech, estruturou as diretrizes da SCTIE.

A partir da SCTIE, foram instituídas algumas estratégias que influenciaram 
a política de C\&T e, consequentemente, as políticas farmacêuticas: o Fórum de Competitividade da Cadeia Produtiva Farmacêutica, que viabilizou a construção da Política Industrial, Tecnológica e de Comércio Exterior, definindo como ações estratégicas aquelas relacionadas a fármacos e medicamentos; e o Programa de Apoio ao Desenvolvimento da Cadeia Produtiva Farmacêutica (Profarma), um programa do Banco Nacional de Desenvolvimento Econômico e Social (BNDES), com financiamento para áreas estratégicas, incluindo a de fármacos. No contexto do Profarma, houve investimentos nos laboratórios farmacêuticos oficiais e a compra da fábrica de medicamentos da GlaxoSmithKline, pela Fiocruz. Este último fato foi alvo de críticas dentro da própria Fiocruz, diante da ausência de um debate mais amplo para discutir tal proposta, conforme revelaram alguns entrevistados da pesquisa. A despeito das críticas, os resultados satisfatórios alcançados pelo Profarma implicaram na sua ampliação, em 2007.

Nesse movimento também ocorreu, em 2004, a II Conferência Nacional de Ciência, Tecnologia e Inovação em Saúde (CNCTIS), possibilitando a elaboração da Política Nacional de Ciência, Tecnologia e Inovação em Saúde (PNCTIS) e a definição da Agenda Nacional de Prioridades de Pesquisa em Saúde. Como desdobramento da PNCTIS, foi criada, em 2006, a Comissão de Incorporação de Tecnologias (Citec), instituindo o primeiro fluxo para a incorporação de tecnologias no SUS, importante estratégia para intermediar as relações entre o Estado e o mercado, na inclusão de produtos no SUS.

Outras disposições (Lei $\mathrm{n}^{\mathrm{o}}$ 10.793/2004

- Lei da Inovação; e Lei no 11.196/2005

- Lei do Bem) foram instituídas, criando

a concessão de incentivos fiscais às empresas que realizassem pesquisa e desenvolvimento de inovação tecnológica. Em 2007, foi regulamentado o Fundo Nacional de Desenvolvimento Científico e Tecnológico, para apoiar financeiramente programas e projetos prioritários nacionais de desenvolvimento.

Também foi criado, por meio do Decreto de 12 de maio de 2008, o Grupo Executivo do Complexo Industrial da Saúde (Gecis), que teve como articulador Reinaldo Guimarães, um militante da RSB, defensor do componente da C\&T na agenda do movimento sanitário, com relevante acúmulo de capacidades técnicas e administrativas. $\mathrm{O}$ Gecis previa o estímulo ao uso do poder de compra do SUS para favorecer a produção, a inovação e a competitividade no Complexo EconômicoIndustrial da Saúde (Ceis). A partir de então, foi estabelecida a lista de produtos estratégicos para o SUS, incluindo farmoquímicos e/ ou medicamentos, além de um conjunto de incentivos fiscais e tributários para condicionar o fortalecimento do setor.

A presença de um governo popular e sua adesão à questão MAF, acrescida da participação de atores militantes do movimento sanitário e envolvidos com essa questão nas estruturas do Ministério da Saúde (MS) não impediram, contudo, que as interferências políticas influenciassem na atuação da SCTIE, que foi alvo da negociação de cargos entre partidos aliados do governo:

[...] houve uma decisão de caráter político e a SCTIE foi reivindicada pelo Partido Progressista, o PP. Assim, houve uma decisão, no âmbito do governo, para que o PP ocupasse uma pasta no Ministério da Saúde. (Norberto Rech).

Apesar disso, as políticas instituídas nesse período promoveram a recuperação e o fomento do setor farmacêutico, compensando o acúmulo histórico da falta de investimentos, ainda que não tenha conferido um dinamismo capaz de situá-lo em condições de competitividade internacional (GADELHA; MALDONADO, 2008). Isto pode ter resultado na amenização dos conflitos existentes na relação entre o mercado farmacêutico e o Estado, na medida em que os interesses do capital passaram a ser processualmente atendidos. 


\section{AS AC̣ÕES DA ANVISA PARA A REGULAC̣ÃO DO MERCADO DE MEDICAMENTOS}

A despeito do distanciamento inicial perante críticas do Partido dos Trabalhadores (PT) quanto ao modelo de agências, houve uma mudança de posicionamento político em relação à Agência Nacional de Vigilância Sanitária (Anvisa) (souto, 2007). A partir de então, foi estabelecido um conjunto de mudanças que implicou maior articulação entre a Anvisa e o MS, provavelmente diante das tendências de regulação impostas pelo processo de globalização e também pela presença de atores do movimento sanitário em cargos de direção.

Entre as ações regulatórias, frisa-se a criação da Câmara de Regulação do Mercado de Medicamentos (CMED) - Lei $\mathrm{n}^{\mathrm{o}} 10.742 / 2003$, um mecanismo para tentar conter os constantes abusos dos preços de medicamentos praticados pelo setor. A Anvisa publicou diversas resoluções, exigindo controles mais rígidos para a produção, a comercialização e o uso de medicamentos, a fim de possibilitar maior segurança e qualidade para o consumidor. Essas medidas regulatórias se constituíram em estratégias para enfrentar os problemas relativos aos riscos relacionados ao uso de medicamentos e outros produtos, que, naquele momento, se avolumavam (ALENCAR, 2016). Ressalta-se o trabalho permanente da Sociedade Brasileira de Vigilância de Medicamentos (Sobravime), que, por meio de seus boletins, fazia o esforço de articular as experiências internacionais às nacionais para promover o debate na área de medicamentos.

Em relação aos medicamentos genéricos, cuja política foi instituída em 1999, pesquisas realizadas pela Anvisa apontavam um crescimento do mercado, indicando que, se anteriormente as indústrias multinacionais e também nacionais foram contrárias à política dos genéricos por entenderem-na como uma ameaça às suas marcas comerciais e também devido aos custos envolvidos, esse posicionamento foi modificado diante das evidências de um mercado crescente. Associada às novas regulamentações para esses medicamentos e à ampliação do poder de compra dos brasileiros, mediante as políticas sociais implantadas nesse período, houve maior consolidação da política de genéricos e do mercado de medicamentos de modo geral.

Outro fato importante refere-se ao processo de discussão da Resolução da Diretoria Colegiada (RDC) no 96/2008, que atualizou a regulação da propaganda de medicamentos. Esse processo teve início em 2005, com a realização da consulta pública, e se manteve por quase três anos, diante dos conflitos entre a defesa dos interesses do setor regulado e da sociedade civil (NASCIMENTO, 2007). Apesar de outras manifestações de organizações da sociedade civil (Instituto de Defesa do Consumidor, Abrasco e Sobravime, entre outras), a regulação da propaganda não sofreu alterações importantes. Ressalte-se que o debate envolvendo a propaganda de medicamentos manteve-se na pauta do movimento sanitário, no sentido de sua proibição total nos meios de comunicação, mas foi mudando a conotação diante dos embates acirrados com o setor regulado.

\section{A CONFERÊNCIA NACIONAL DE MEDICAMENTOS E ASSISTÊNCIA FARMACÊUTICA E A POLÍTICA NACIONAL DE ASSISTÊNCIA FARMACÊUTICA}

O debate sobre a necessidade da realização da conferência temática sobre medicamentos e assistência farmacêutica já existia no âmbito do Conselho Nacional de Saúde (CNS), motivado pela CPI de medicamentos, ocorrida em 1999, e pela presença de atores vinculados à questão MAF no Estado, que defendiam essa pauta. Inclusive, essa já havia sido uma recomendação das IX, X e XI Conferências de Saúde, e da Conferência Nacional de Vigilância Sanitária (Conavisa). Porém, houve dificuldades dentro do próprio CNS, por parte de representantes 
do governo, para que a Conferência Nacional de Medicamentos e Assistência Farmacêutica (CNMAF) ocorresse, conforme revela a análise das atas das reuniões do CNS.

Foi estabelecido, portanto, um jogo político para garantir a realização da CNMAF com abordagem temática mais ampla e não apenas restrita ao componente técnico do medicamento. E também para que a coordenação da conferência fosse composta por alguém mais próximo do CNS e das organizações farmacêuticas. Os dois principais nomes (Jorge Bermudez e Clair Castilho) à coordenação da conferência eram atores políticos importantes, com envolvimento na questão MAF, mas foi escolhida a conselheira do CNS, Clair Castilho, dadas as suas vinculações profissionais com atores que ocupavam a SCTIE e o Departamento de Assistência Farmacêutica (DAF). Em setembro de 2003, a conferência foi realizada.

Muitas proposições da CNMAF integraram a Política Nacional de Assistência Farmacêutica (PNAF), que foi aprovada em reunião do CNS, pela Resolução $n^{\circ} 338 / 2004$. Entende-se, portanto, que a PNAF foi resultante de um processo de construção coletiva, fundamentada no controle social, diferente do modo como se deu a construção da PNM, que, apesar de ter tido a participação do CNS, foi predominantemente organizada por um grupo técnico decidido pelo MS, conforme revela a análise de documentos e das falas dos entrevistados. A resolução estabeleceu que a PNAF fosse integrante da Política Nacional de Saúde, coerente com os princípios do SUS e norteadora para a formulação de outras políticas setoriais, a exemplo da C\&T, do Desenvolvimento Industrial e da Formação de Recursos Humanos.

\section{A REORIENTAC̣ÃO DA ASSISTÊNCIA FARMACÊUTICA NO SUS}

Após a instituição da PNAF, o processo de reorientação da assistência farmacêutica foi viabilizado com a implantação do Pacto pela
Saúde e com a Portaria no 698/2006, que definiu as responsabilidades das três esferas de gestão nas ações de saúde, estabelecendo blocos de financiamento, incluindo o de assistência farmacêutica. Este último foi posteriormente excluído, com a publicação da Portaria $n^{0}$ 204/2007, tornando mais difícil a efetuação das ações de assistência farmacêutica nos serviços. Até então, essas ações eram restritas à disponibilização de medicamentos e, apesar de haver desperdícios e outros prejuízos, por motivos diversos, não havia consenso, entre os gestores, de que os serviços farmacêuticos poderiam resultar, também, em implicações econômicas favoráveis à saúde (ALENCAR; NASCIMENTO, 2011).

Esses aspectos fizeram com que o DAF disponibilizasse uma ferramenta de inovação tecnológica para a gestão das informações do setor, o Hórus - Sistema Nacional de Gestão da Assistência Farmacêutica - e as 'Diretrizes para estruturação das farmácias no âmbito do SUS', que tratava de recomendações das condições (instalações, equipamentos, boas práticas, gerenciamento dos produtos e atividades clínicas) mínimas necessárias para a qualificação das atividades. Ambos, com desafios para sua implementação nos serviços (COSTA; NASCIMENTO JUNIOR, 2012).

Outra iniciativa importante deu-se com a criação dos Núcleos de Apoio à Saúde da Família (Nasf), por meio da Portaria $\mathrm{n}^{\circ}$ $154 / 2008$, que previa a inclusão de outros profissionais para ampliar as ações de atenção básica. Novas mobilizações foram desenvolvidas, envolvendo Conselhos Regionais e outras organizações para a tarefa de convencer os gestores a incorporarem os farmacêuticos nas equipes Nasf.

Em paralelo a esse movimento para a inserção da assistência farmacêutica no SUS, também havia outro, em torno da defesa da farmácia como estabelecimento de saúde. Essas questões foram articuladas como objeto de pauta política na Frente Parlamentar em Defesa da Assistência Farmacêutica, lançada em 2008, criando condições para 
que tal pauta ganhasse espaço no âmbito do Parlamento. Apesar de se propor defensora da assistência farmacêutica, em algumas situações, a Frente Parlamentar pareceu preservar mais os interesses corporativistas do que os da pauta coletiva. Além disso, fatos posteriores indicaram conflitos de interesses entre seus integrantes, já que alguns receberam apoio financeiro de grandes empresas farmacêuticas em campanhas eleitorais.

\section{POLÍTICAS PARA A AMPLIAÇÃO DO ACESSO A MEDICAMENTOS}

Política importante para a ampliação do acesso aos medicamentos refere-se ao Programa Farmácia Popular do Brasil (PFPB). Havia um Projeto de Lei (PL) $n^{\circ}$ 4.702/1994, sobre farmácias populares, de autoria do deputado federal Eduardo Jorge (PT do estado de São Paulo), que oportunamente foi identificado, durante o período de campanha presidencial, pela equipe do candidato Lula, como interessante à proposta de campanha. A partir daí, a ideia da criação de farmácias populares foi inserida na campanha de governo do presidente Lula e, após a sua vitória, se tornou uma prioridade a ser implementada pela equipe do MS, ainda que não houvesse um consenso quanto a ela dentro da própria equipe que se responsabilizaria por sua execução.

Esse assunto foi comentado sem detalhamentos no CNS, no momento da discussão da PNAF, conforme pode ser evidenciado em ata de reunião, e representantes do setor farmacêutico foram convidados para discutir a proposta desse programa. Contudo, não houve um debate coletivo, o que gerou posicionamentos contrários entre os envolvidos com a questão MAF, inclusive na Fiocruz, que teria responsabilidade fundamental na instituição do programa.

Entre consensos e contradições, o PFPB foi instituído na modalidade rede própria, por meio do Decreto ${ }^{\circ}$ 5.090/2004 (regulamentando a Lei $\mathrm{n}^{\mathrm{o}}$ 10.858/2004), esclarecendo que estava sendo criado visando à universalização do acesso a medicamentos e à diminuição do impacto dos gastos com medicamentos no orçamento familiar, para os cidadãos assistidos pela rede privada. Parece ter havido, portanto, uma preocupação em deixar clara a não associação do PFPB à dispensação de medicamento já realizada pelo SUS, haja vista as críticas de que o programa apresentava inconstitucionalidades ao fornecer medicamentos mediante copagamento, além do argumento de que as farmácias do SUS ficariam prejudicadas.

Dois anos após a sua implantação, o PFPB foi ampliado para as farmácias da rede privada (modalidade 'Aqui tem Farmácia Popular'), conforme já estava previsto no Decreto $\mathrm{n}^{\mathrm{o}} 5.090 / 2004$, estabelecendo que os cidadãos poderiam adquirir medicamentos para o tratamento de hipertensão arterial e diabetes nas farmácias privadas, mediante complementação da diferença do preço de venda (determinado conforme critérios definidos pela CMED). O modelo também gerou muitas críticas sob o argumento de que o Estado estimularia o mercado farmacêutico em vez de fortalecer as unidades de saúde do SUS, alvo de constantes queixas de indisponibilidade de medicamentos. As entrevistas realizadas revelaram posicionamentos divergentes entre sanitaristas militantes do movimento sanitário. Enquanto uns entendiam o modelo de parceiras com as farmácias privadas como mais racional e viável para a garantia do acesso aos medicamentos do que o modelo de rede própria, outros consideravam ambos os modelos um retrocesso no processo da RSB. A despeito das críticas, até o final do período Lula, houve expansão significativa do número de usuários, em ambas as modalidades (SILVA; CAETANO, 2015).

A estruturação das ações de assistência farmacêutica alcançada com a criação da SCTIE, do DAF e da Citec para adequar os processos de incorporação de tecnologias não foi suficiente para conter o avanço do fenômeno da judicialização protagonizado 
pela indústria da saúde, especialmente a indústria farmacêutica. Um movimento por parte das secretarias estaduais de saúde passou a ser desenvolvido no sentido de definir estratégias para o enfrentamento dessa questão. Foram realizadas parcerias das secretarias estaduais com Procuradorias Públicas, ações junto aos pacientes, debates temáticos e pesquisa. Houve, também, reações no âmbito do Legislativo. O senador Tião Viana (PT do estado do Acre) elaborou o Projeto de Lei ${ }^{0}$ 219/2007, prevendo alterações na Lei ${ }^{\circ}$ 8.080/1990, para dispor sobre a oferta de procedimentos terapêuticos e a dispensação de medicamentos no âmbito do SUS, delimitando critérios para o atendimento do princípio da integralidade.

No âmbito do Executivo, o MS regulou, por meio da Portaria ${ }^{\circ} 2.981 / 2009$, o componente especializado da assistência farmacêutica, incluindo os medicamentos de custo mais elevado, que compunham o principal objeto das demandas judiciais, e estabelecendo que a integralidade do tratamento medicamentoso deveria ser orientada por Protocolos Clínicos e Diretrizes Terapêuticas (PCDT). Apesar dessas disposições, houve ampliação das demandas judiciais (souZA ET AL., 2017; TERRAZAS, 2014)

Também na perspectiva de assegurar o acesso aos medicamentos, fato político relevante diz respeito às ações relativas aos antirretrovirais: após intensas negociações com o laboratório Merck Sharp \& Dohme para a redução do preço do medicamento Efavirenz, o presidente Lula decretou, em maio de 2007, seu licenciamento compulsório. Com essa medida, o Brasil passou a importar o genérico do Efavirenz de laboratórios qualificados pela Organização Mundial da Saúde (OMS) e, a partir de 2009 , passou a ser produzido pelos laboratórios oficiais Farmanguinhos/ Fiocruz e Lafepe, em cooperação com um consórcio de empresas privadas formado pela Globe Química, pela Cristália e pela Nortec Química (DOMINGUEZ, 2009). Naquele momento, a Anvisa teve função importante como articuladora das etapas produtivas e de regulação do registro do medicamento, conferindo agilidade ao processo.

A determinação do licenciamento compulsório possibilitou não apenas a obtenção do medicamento Efavirenz por um preço reduzido, como também produziu impulso para processos de transferência de tecnologia de um fármaco relevante para as políticas de saúde, iniciou um movimento para a diminuição da dependência do Brasil em relação ao mercado farmacêutico mundial e fortaleceu a participação nacional no complexo industrial da saúde.

\section{POLÍTICAS PARA A INSERC̣̃̃ DAS TERAPIAS COMPLEMENTARES E ALTERNATIVAS NO SUS}

Consoante às recomendações destacadas durante as CNS, a Conavisa, ocorrida em 2001, e também nas CNCTS e CNCTIS, foram retomados os debates relativos à utilização do potencial da flora brasileira para a pesquisa de fármacos e pela inclusão da fitoterapia, da homeopatia e de outras práticas complementares no SUS. Os debates em torno dessa pauta antecederam o período Lula, mas somente nesse governo foram instituídas a Política Nacional de Práticas Integrativas e Complementares no SUS (PNPICS), bem como a Política e o Programa Nacional de Plantas Medicinais e Fitoterápicos. Salientase, ainda, a implantação da Relação Nacional de Plantas Medicinais de Interesse do SUS (Renisus), na qual constam as plantas medicinais que apresentam potencial para gerar produtos de interesse do SUS.

A implantação de tais políticas só foi viabilizada a partir da reestruturação da assistência farmacêutica no âmbito do MS e de uma conjuntura mais favorável ao setor de C\&T para o País, na qual foi retomada a necessidade de discutir e efetuar ações direcionadas à valorização da biodiversidade brasileira, por meio do fomento à pesquisa e ao desenvolvimento de fitoterápicos. Também foi determinante a participação de 
diferentes organizações da sociedade civil, inclusive de entidades farmacêuticas como a Fenafar e o Conselho Federal de Farmácia, além do Conselho Nacional de Secretarias Municipais de Saúde (Conasems), do CNS e de organizações de representantes de práticas complementares.

\section{Período Dilma (2011-2014)}

\section{A REORIENTAC̣ÃO E A QUALIFICAC̣ÃO DA ASSISTÊNCIA FARMACÊUTICA NO SUS}

Resultante de um processo de discussão que já vinha acontecendo no Legislativo e no Judiciário, foi instituída a Lei ${ }^{\circ}$ 12.401/2011 (regulamentada pelo Decreto $\mathrm{n}^{\mathrm{o}} 7.646 / 2011$ ), tendo como objeto o estabelecimento de critérios para a oferta de procedimentos terapêuticos e a dispensação de medicamentos no âmbito do SUS. Essas disposições estabeleceram a Comissão Nacional de Incorporação de Tecnologias (Conitec), que incorporou e aprimorou as funções da Citec, assumindo nova composição, mas foi alvo de críticas, também relatadas por entrevistados, ao excluir a representação das sociedades científicas e das universidades.

Com a publicação do Decreto $n^{\circ}$ $7.508 / 2011$, foram previstas mudanças para a assistência farmacêutica, definindo-se instrumentos para orientar o processo de incorporação de tecnologias e a regulação do acesso a medicamentos no SUS. Essas medidas geraram expectativas de amenização de conflitos impostos pelo Judiciário. Porém, persistiram outros, decorrentes da prática da prescrição médica e da não obediência aos dispositivos legais e aos processos de incorporação de tecnologias estabelecidos pela Conitec.

Visando à continuidade da reorientação da assistência farmacêutica, foi instituído, por meio da Portaria ${ }^{\circ} 1.214 / 2012$, o Programa Nacional de Qualificação da Assistência Farmacêutica (Qualifar-SUS). Estruturado em quatro eixos (estrutura, educação, informação e cuidado), o programa fundamentou-se em diretrizes que previam, entre outras ações, ampliar a disponibilidade de medicamentos e promover condições favoráveis para a estruturação dos serviços farmacêuticos no SUS. Indicava, portanto, perspectivas de mudanças para a área. Apesar do aparente êxito dessa iniciativa, diante da realização de diferentes edições até 2014, ainda não foram identificados estudos que tenham avaliado seus resultados e implicações para a assistência farmacêutica no SUS.

Passados 25 anos da implementação do SUS, e mais de 10 anos das principais políticas farmacêuticas (PNM, Anvisa, genéricos, PNAF e PFPB), a avaliação realizada pelo MS (BRASIL, 2014A), por meio de um cotejamento das propostas elencadas durante a I CNMAF, ocorrida em 2004, com as ações implementadas no decorrer de 10 anos, apontou que: houve cumprimento de grande parte das propostas aprovadas na CNMAF; consolidou-se o direito de acesso a medicamentos; os componentes da assistência farmacêutica e o PFPB foram firmados como estratégias para promover o acesso; e houve a ampliação do financiamento de medicamentos. Assim, também houve uma consolidação da PNAF como política de saúde, articulando-se com outras políticas, particularmente com a de medicamentos, de C\&T, de Desenvolvimento Industrial e de Formação de Recursos Humanos.

Os dez anos da PNAF também foram marcados por mobilizações em torno da pauta da farmácia como estabelecimento de saúde, um movimento que já acumulava 20 anos de luta, decorrente da tramitação de projetos de lei com proposições divergentes quanto a aspectos sanitários. Após intensas negociações entre entidades representativas da categoria farmacêutica, parlamentares e empresários do setor, foi promulgada a Lei $\mathrm{n}^{\mathrm{o}} 13.021 / 2014$, que definiu farmácia como unidade de serviços destinada a prestar assistência farmacêutica, assistência à saúde e orientação sanitária individual e coletiva 
(BRASIL, 2014B). Ainda que esta medida seja positiva e necessária, a realidade mostra que ela tem pouca aplicabilidade prática, pois encontra divergência nos interesses do setor privado (tanto que foi modificada em alguns aspectos, por meio da Medida Provisória ${ }^{\circ}$ 653/2014) e nas limitações diversas da esfera pública, a despeito de todos os esforços despendidos para aprimorar a assistência farmacêutica no SUS.

\section{MUDANÇAS E DESAFIOS PARA A CONTINUIDADE DO PFPB}

Em 2011, foi realizada ampliação do PFPB, e instituída, por meio da Portaria ${ }^{\circ} 184 / 2011$, a campanha 'Saúde não tem preço', que passou a disponibilizar medicamentos para patologias específicas (inicialmente, para hipertensão e diabetes e, em seguida, para asma) sem custo adicional (isentos de copagamento), nas farmácias de unidade própria e naquelas conveniadas ao 'Aqui tem Farmácia Popular'.

Após 10 anos de implantação, o PFPB acumulava resultados positivos: foi identificado como o programa social do governo federal mais bem avaliado pela população brasileira; articulou-se a outros programas do governo, a exemplo do Plano Brasil Sem Miséria, do Programa Brasil Carinhoso, do Plano de Ações Estratégicas para o Enfrentamento das Doenças Crônicas Não Transmissíveis e do Programa Mais Médicos; registrou-se maior acesso aos medicamentos para doenças crônicas (com destaque para hipertensão, diabetes e asma), especialmente após a instituição do 'Saúde não tem preço'; e foi referido pela Pesquisa Nacional de Saúde 2013 como uma política que contribuiu para o acesso aos medicamentos pela população (IBGE, 2014). Além disto, é um programa com visibilidade internacional, referido pela OMS como exemplo bem sucedido para a ampliação do acesso a medicamentos (WHO, 2012).

Apesar dos resultados alcançados, o programa não esteve isento de polêmicas, particularmente relacionadas à modalidade 'Aqui tem Farmácia Popular', que foi, inclusive, objeto de auditoria do Tribunal de Contas da União, em 2011. Além disto, estudos têm apontado críticas importantes ao programa, especialmente quanto à sua capacidade de articular outras políticas farmacêuticas (YAMAUTI; BARBERATO-FILHO; LOPES, 2015).

Ainda que os dispositivos legais norteadores do programa estabeleçam um conjunto de exigências sanitárias, particularmente a respeito de estrutura e presença de farmacêutico durante o horário de funcionamento das farmácias da rede própria ou credenciada, os estudos recentes realizados (SILVA; CAETANO, 2015), inclusive os resultados divulgados pelo próprio MS (BRASIL, 2014B), não apontam qualquer ênfase quanto à qualidade dos serviços dispensados nessas farmácias. Apesar da tendência de ampliação do programa, particularmente por meio do credenciamento de farmácias privadas ao 'Aqui tem Farmácia Popular', foi evidente uma inflexão, nos últimos anos, inclusive com reflexos na modalidade rede própria, havendo fechamento de unidades em todo o País.

\section{REGULAC̣ÃO SANITÁRIA DE MEDICAMENTOS: CONFLITOS E ESTRATÉGIAS}

Fato marcante no âmbito da regulação sanitária refere-se ao caso dos medicamentos anorexígenos anfetamínicos (Anfepramona, Femproporex, Mazindol) e da Sibutramina - utilizados clinicamente como inibidores de apetite -, que trouxeram para o debate a real autonomia da Anvisa. O posicionamento inicial da agência foi proibir a comercialização de todos os produtos anorexígenos. Contudo, houve posições divergentes, por parte de organizações médicas e farmacêuticas, que foram contrárias à proibição dos anorexígenos, defendendo como suficiente o maior controle. Por outro lado, entidades de saúde - Sobravime, Abrasco, Centro Brasileiro de Estudos de Saúde (Cebes), Escola Nacional de Saúde Pública Sergio Arouca (Ensp)/Fiocruz, Instituto Nacional 
de Controle de Qualidade em Saúde (INCQS)/Fiocruz etc. - apoiaram a Anvisa.

Após as discussões, a Anvisa decidiu, por meio da RDC $n^{\circ} 52 / 2011$, pela proibição do uso dos anfetamínicos (Anfepramona, Femproporex e Mazindol), mas pela continuidade da comercialização da Sibutramina, com o estabelecimento de medidas mais rígidas de controle para sua prescrição e dispensação. No jogo político e econômico envolvido na tomada de decisão da Anvisa, não se pode desprezar a informação de que o Brasil respondia por mais da metade das vendas de Sibutramina no mundo, tampouco os embates já acumulados entre o governo e a categoria médica, diante da criação do Programa Mais Médicos, em 2013, ou o veto da presidente Dilma ao Ato Médico, tendo em vista as eleições de 2014.

Esses embates não finalizaram, e a pauta continuou na agenda do Legislativo, visando sustar os efeitos da referida RDC, inicialmente por meio do PL ${ }^{\circ}$ 2.431/2011, de autoria do deputado federal e empresário Felipe Bornier - Partido Social Democrático, do Estado do Rio de Janeiro. Ao final da tramitação, foi promulgado o Decreto Legislativo $n^{\circ} 273 / 2014$, sustando os efeitos da RDC Anvisa $n^{\circ}$ 52/2011. A Anvisa reagiu aprovando normas mais rigorosas por meio da RDC n ${ }^{\circ} 50 / 2014$, mas a pauta esteve sob tensões de possíveis novas interferências. Tanto que, recentemente, foi aprovada a Lei $\mathrm{n}^{\mathrm{o}} 13.454 / 2017$, autorizando a produção, a comercialização e o consumo, sob prescrição médica, dos referidos anorexígenos.

\section{AS PARCERIAS DE DESENVOLVIMENTO PRODUTIVO E O PROCESSO DE REVISÃO DA LEI DE PATENTES}

As políticas do Ceis instituídas encontraram condições de serem continuadas e fortalecidas, sendo incorporadas ao Plano Brasil Maior (a Política Industrial, Tecnológica e de Comércio Exterior do governo federal), que visa fortalecer a indústria brasileira, dando maior autonomia ao País em relação à produção de tecnologias estratégicas ao SUS. Nessa perspectiva, as Parcerias para Desenvolvimento Produtivo (PDP) tiveram grande impulso, sendo estabelecido um primeiro marco regulatório (Portaria $\mathrm{n}^{\mathrm{o}}$ 837/2012), mediante acordos de cooperação entre instituições (públicas e privadas), para desenvolvimento, transferência, absorção de tecnologia e capacitação produtiva do País em produtos estratégicos para o SUS.

$\mathrm{O}$ fato de o Brasil dispor de uma rede de laboratórios públicos foi determinante para viabilizar o cumprimento dos dispositivos constitucionais relativos à C\&T e ao o estabelecimento das PDP:

[...] a existência de uma rede de laboratórios oficiais sempre teve grande importância. Tanto é que a política da PDP só foi possível fazer com a parceria dos laboratórios públicos. (José Gomes Temporão).

De acordo com dados do MS, até setembro de 2014, foram formalizadas 104 parcerias (envolvendo 57 laboratórios privados e 19 laboratórios púbicos oficiais), que resultaram em 101 produtos (sendo 66 medicamentos, 7 vacinas - 6 já são adquiridas pelo Programa Nacional de Imunizações, 27 produtos para saúde e 5 projetos de pesquisa e desenvolvimento). Desses produtos, 29 já possuem registro na Anvisa, dos quais 9 são adquiridos pelo DAF (BRASIL, 2014A).

Dada a relevância que a política industrial da saúde assumiu para o Estado brasileiro, houve atualização do marco regulatório das PDP, estabelecido por meio da Portaria ${ }^{\circ}$ $2.531 / 2014$. O novo marco também foi motivado por denúncias e investigações envolvendo o laboratório Labogen S/A Química Fina e Biotecnologia na Operação Lava Jato da Polícia Federal, iniciada em março de 2014 para investigar um esquema bilionário de desvio e lavagem de dinheiro envolvendo a Petróleo Brasileiro S/A (Petrobras), diante de possíveis irregularidades nos contratos, 
com suspeita de envolvimento de propinas para a execução do acordo.

Apesar do potencial que vem sendo identificado por meio das PDP, essa estratégia divide opiniões, entre sanitaristas, sob o argumento de possíveis riscos e limitações inerentes à relação público-privada, exigindo que outras estratégias sejam pensadas entre elas, a revisão da legislação de patentes - para garantir a sustentabilidade do SUS. Nesse sentido, um debate sobre essa pauta tem ocorrido, desde 2011, na Câmara dos Deputados, por meio do Centro de Estudos e Debates Estratégicos, resultando na publicação 'A revisão da Lei de Patentes: inovação em prol da competitividade nacional', que serviu de base para o Projeto de Lei $n^{\circ}$ $5.402 / 2013$, em tramitação, de autoria dos deputados Newton Lima - PT, do Estado de São Paulo - e Dr. Rosinha - PT, do Estado do Paraná -, propondo, entre outros aspectos: limitar a duração do prazo das patentes (de 20 para 10 anos); alterar o rigor dos critérios de patenteabilidade; modificar o dispositivo sobre a anuência prévia da Anvisa; e instituir o mecanismo do uso público não comercial.

Também no âmbito do poder Legislativo, há outro movimento importante para a área de $\mathrm{C} \& \mathrm{~T}$ referente à promulgação da EC n ${ }^{\circ} 85 / 2015$, resultante da Proposta de Emenda Constitucional (PEC) $n^{0} 290 / 2013$, de autoria da deputada Margarida Salomão - PT, do Estado de Minas Gerais -, que altera e adiciona dispositivos na Constituição para atualizar o tratamento das atividades de ciência, tecnologia e inovação.

\section{Discussão}

No período analisado, a participação de intelectuais orgânicos vinculados ao movimento sanitário e à questão MAF no âmbito do Estado, na busca por hegemonia, oportunizou a produção de fatos políticos que resultaram em políticas farmacêuticas consoantes ao projeto da RSB, representado no relatório da VIII CNS, conforme examinado por Paim (2008). Destaca-se, neste processo, a articulação de aspectos relativos à oferta e à disponibilidade de medicamentos aos componentes industrial, tecnológico, dos serviços farmacêuticos, das práticas integrativas e do uso racional.

Vale comentar que a pauta de medicamentos era componente importante na campanha de governo (2002) do então candidato Lula, ainda que sob o aspecto mercadológico-político, que se expressou principalmente no PFPB. Esse, por sua vez, diferente do conceito de estratégia programática proposto por Testa (1995), foi criado por uma decisão política e não técnica, o que pode auxiliar na compreensão da descontinuidade que caracteriza o programa na conjuntura atual, na qual a modalidade rede própria foi finalizada por decisão do Executivo, apesar de protestos da sociedade.

As políticas instituídas nesse período se inseriram na perspectiva de expansão do consumo, existente em outras políticas sociais do Governo Lula, para favorecer o desenvolvimento econômico. A criação de condições para a ampliação do consumo de medicamentos também estendeu a necessidade de produção, mantendo-se, assim, o ciclo produtivo econômico (MARX, 1996) da saúde no País. Esse estímulo ao consumo foi captado pelo mercado como oportunidade para produzir novas necessidades sociais (HELLER, 1986) e assegurar a manutenção do ciclo produtivo econômico por meio de sua força expansionista, com implicações para o Estado. As demandas judiciais se ampliaram nesse contexto, quando o mercado identificou as fragilidades do Estado para assegurar e regular esse direito ao medicamento (como necessidade de existência), firmado na Constituição e na Lei no 8.080/1990.

Particularmente no período Dilma, houve continuidade das políticas instituídas, ampliando as ações relativas à $\mathrm{C} \& \mathrm{~T}$ e de acesso aos medicamentos, mas com dificuldades para maiores avanços diante da conjuntura 
econômica e da intensificação dos conflitos na relação Estado-mercado-sociedade. Estes conflitos têm sido ampliados proporcionalmente à interferência dos interesses do capital, constatada, por exemplo, nos debates de normas sanitárias, como na regulação da propaganda de medicamentos e dos fármacos anorexígenos. Isto indica que, apesar do movimento de mudança que caracterizou o período analisado, tem havido predominância da dimensão econômica em detrimento da dimensão sanitária relativa à questão MAF.

\section{Conclusões}

A análise dos fatos produzidos nesse período, bem como a identificação dos atores envolvidos, dos conflitos e das estratégias utilizadas evidencia um momento de avanços na instituição de políticas farmacêuticas, com conquistas significativas, ao tomar como referência a RSB. Contudo, o melhor entendimento deste processo deve estar amparado nos fatos políticos antecedentes relativos à questão MAF e à RSB, aspectos estes examinados amplamente na pesquisa que originou o presente artigo.
Considerando que a conjuntura atual é caracterizada pela permanência e pela ampliação de conflitos, há necessidade de condução de novos processos, visando produzir uma relação de forças que seja capaz de evitar a continuidade de perdas de conquistas alcançadas e de elaborar meios de enfrentamento, a fim de garantir o direto à saúde e à assistência farmacêutica, conforme previsto na Constituição, e coerente ao projeto de transformação social defendido pelo movimento sanitário.

\section{Colaboradores}

Os autores participaram de todas as etapas, incluindo a concepção, a interpretação dos dados, a elaboração do texto e a revisão final do artigo.

\section{Agradecimentos}

Ao Conselho Nacional de Desenvolvimento Científico e Tecnológico (CNPq), ao MS e ao Observatório de Análise Política em Saúde. 


\section{Referências}

ALENCAR, T. O. S. A Reforma Sanitária Brasileira e a questão medicamentos/assistência farmacêutica. 2016. 439 f. Tese (Doutorado em Saúde Pública) Universidade Federal da Bahia, Salvador, 2016.

ALENCAR, T. O. S.; NASCIMENTO, M. A. A. Assistência Farmacêutica no Programa Saúde da Família: encontros e desencontros do processo de organização. Ciência \&t Saúde Coletiva, Rio de Janeiro, v. 16, n. 9, p. 3939-3949, set. 2011.

BARDIN, L. Análise de conteúdo. 7. ed. São Paulo: Edições 70, 2011.

BRASIL. Ministério da Saúde. Os 10 anos da Política Nacional de Assistência Farmacêutica: PNAF. Brasília, DF: Ministério da Saúde, 2014a. Disponível em: <http:// portalsaude.saude.gov.br/images/pdf/2015/janeiro/08/ Avaliacao-PNAF.pdf>. Acesso em: 2 jan. 2016.

Ministério da Saúde. Os 10 anos do Programa Farmácia popular do Brasil. Brasília, DF: Ministério da Saúde, 2014b.

CORDEIRO, H. A política de medicamentos. In: GUIMARÃES, R. (Org.). Saúde e medicina no Brasil: contribuição para um debate. Rio de Janeiro: Edições Graal, 1978, p. 253-265.

COSTA, K. S.; NASCIMENTO JÚNIOR, J. M. HÓRUS: inovação tecnológica na assistência farmacêutica no sistema único de saúde. Revista de Saúde Pública, São Paulo, v. 46, p. 91-99, 2012.

COUTINHO, C. N. O leitor de Gramsci. Rio de Janeiro: Civilização Brasileira, 2011.

DOMINGUEZ, B. Passos decisivos em produção e inovação. Radis, Rio de Janeiro, n. 78, p. 11-13, fev. 2009. Disponível em: <http://www6.ensp.fiocruz.br/radis/ revista-radis/78/reportagens/passos-decisivos-em-producao-e-inovacao>. Acesso em: 2 fev. 2017.

GADELHA, C. A. G.; MALDONADO, J. M. S. V. O papel da inovação na indústria farmacêutica: uma janela de oportunidade no âmbito do complexo industrial da saúde. In: BUSS, P. M.; CARVALHEIRO, J. R.; CASAS, C. P. R. (Org.). Medicamentos no Brasil: inovação e acesso. Rio de Janeiro: Fiocruz, 2008. p. 41-59.

HELLER, A. A teoria das necessidades em Marx. Barcelona: Península, 1986.

\section{INSTITUTO BRASILEIRO DE GEOGRAFIA E} ESTATÍSTICA (IBGE). Pesquisa Nacional de Saúde: 2013. Rio de Janeiro: IBGE, 2014. Disponível em: <ftp:// ftp.ibge.gov.br/PNS/2013/pns2013.pdf>. Acesso em: 12 nov. 2015.

LEDOGAR, R. J.; KUCINSKI, B. Fome de lucros: atuação das multinacionais de alimentos e remédios na América Latina. São Paulo: Brasiliense, 1977.

MARX, K. Introdução à crítica da Economia Política In: MARX, K. Os pensadores. São Paulo: Nova Cultural, 1996, p. 25-54.

MATUS, C. Teoria do jogo social. São Paulo: Fundap, 2005 .

NASCIMENTO, A. C. Propaganda de medicamentos: É possível regular? 2007. 286 f. Tese (Doutorado em Saúde Coletiva) - Universidade do Estado do Rio de Janeiro, Rio de Janeiro, 2007.

PACHECO, M. V. Indústria farmacêutica e segurança nacional. Rio de Janeiro: Civilização Brasileira, 1968.

PAIM, J. S. Reforma Sanitária Brasileira: contribuições para compreensão e crítica. Salvador: EDUFBA; Rio de Janeiro: Fiocruz, 2008.

SILVA, R. M.; CAETANO, R. Programa Farmácia Popular do Brasil caracterização e evolução entre 2004-2012. Ciência e Saúde Coletiva, Rio de Janeiro, v. 20, n. 10, p. 2943-2956, out. 2015.

SOUTO, A. C. Processo de gestão na Agência Nacional de Vigilância sanitária - Anvisa. 2007. 191 f. Tese (Doutorado em Saúde Pública) - Instituto de Saúde 
Coletiva, Universidade Federal da Bahia, Salvador, 2007.

SOUZA, L. E. P. F. et al. Acompanhamento das decisões judiciais relativas à saúde. In: OBSERVATÓRIO DE ANÁLISE POLÍTICA EM SAÚDE, 2017, Salvador. Relatório Acompanhamento de Políticas. Salvador: Ministério da Saúde, 2017. Disponível em: <http:// www.analisepoliticaemsaude.org/oaps/matriz/analises/1/>. Acesso em: 8 jul. 2017.

TEIXEIRA, C. F.; PAIM, J. S. A política de saúde no governo Lula e a dialética do menos pior. Saúde em Debate, Rio de Janeiro, v. 29, n. 71, p. 268-283, set./dez. 2005. Disponível em: <https://repositorio.ufba.br/ri/ handle/ri/6037>. Acesso em: 2 fev. 2017.

TERRAZAS, F. Novos elementos no cenário da judicialização da saúde: análise das decisões dos tribunais superiores. In: SANTOS, L.; TERRAZAS, F. Judicialização da Saúde no Brasil. Campinas: Saberes, 2014.

TESTA, M. Pensamento estratégico e lógica de programação: o caso da saúde. São Paulo: Hucitec; Rio de Janeiro: Abrasco, 1995.
. Vida. Senãs de Identidad (Miradas al Espejo).

Salud Colectiva, Buenos Aires, v. 1, n. 1, p. 33-58, 2005.

WORD HEALTH ORGANIZATION (WHO). The

Pursuit of Responsible Use of Medicines: Sharing and

Learning from Country Experiences. [S. 1.]: WHO,

2012. Disponível em: <http://apps.who.int/iris/bits-

tream/10665/75828/1/WHO_EMP_MAR_2012.3_eng. pdf>. Acesso em: 10 jan. 2016.

\section{YAMAUTI, S. M.; BARBERATO-FILHO, S.; LOPES,}

L. C. Elenco de medicamentos do Programa Farmácia Popular e a Política Nacional de Assistência Farmacêutica. Cadernos de Saúde Pública, Rio de Janeiro, v. 31, n. 8, p. 1648-1662, ago. 2015.

Recebido para publicação em junho de 2017

Versão final em agosto de 2017

Conflito de interesses: inexistente

Suporte financeiro: Pesquisa vinculada ao Projeto Análise de

Políticas de Saúde no Brasil (2003-2017), apoiado pelo CNPq e Ministério da Saúde (Chamada MCTI / CNPq / CT-Saúde / MS / SCTIE / Decit N 41/2013) 